\title{
Index to Volume 125
}

\section{Compiled by Leslie Cody}

Abies balsamea, 58,107,237,298,359

lasiocarpa, 7,232

Abietinella abietina, 332

Abolit, D. and J. Gilhen. Eastern Chipmunk, Tamias striatus, Attack on a Maritime Garter Snake, Thamnophis sirtalis pallidulus, at Cooks Lake, Halifax County, Nova Scotia, Canada, 55

Acer rubrum, 58,237,359 saccharum, $58,107,237,363$

Achillea millefolium, 204,303 millefolium var. occidentalis, 201

Achnatherum sp., 317 hymenoides, 349

Aconitum delphiniifolium, 230

Acorn, J., 373

Actaea pachypoda, 237

Adropion cf. prorsirostre, 24

Agalinis neoscotica, 207

Agelaius phoeniceus, 258

Aglais milberti milberti, 302

Agrimonia gryposepala, 237

Agrimony, Tall, 237

Agropyron cristatum, 133,350

Agrostis mertensii, 241 stolonifera, 63,204

Aitken, R.J., 235

Alaska, Butterflies Recorded on Flattop Mountain, Anchorage, 357

Alberta, Canada: A Preliminary Survey, Tardigrades of Fish Creek Provincial Park, 22

Alberta, Canada, Fetid Dogweed (Dyssodia papposa; Asteraceae) and Slender Russian Thistle (Salsola collina; Amaranthaceae), New to, 366

Alberta, Seasonal Moulting in Deer Mice (Peromyscus maniculatus) in the Rocky Mountains, 126

Alberta, Some Observations on the Pollination of RoundLeaf Orchid, Galearis (Amerorchis) rotundifolia, Near Jasper, 47

Alces alces, 194,353

Alder, Speckled, 58,359

Alliaria petiolata, 249

Allocyttus verrucosus, 74

Allocyttus verrucosus New to the Fish Fauna of Canada, The Warty Dory, 74

Alnus incana, 58 incana rugosa, 359

Amaranthus caudatus, 338 retroflexus, 338

Ambloplites rupestris, 16

Amblyscirtes vialis, 302

Ambophthalmus sp., 376 eurystigmatephoros, 376

Ambrosia artemisiifolia, 63

Ameiurus nebulosus, 18

Amelanchier alnifolia, 300 alnifolia var. compacta, 299
American Society of Mammalogists Annual Meeting 2012, 181

Amerorchis rotundifolia, 47

Amerorchis) rotundifolia, Near Jasper, Alberta, Some Observations on the Pollination of Round-Leaf Orchid, Galearis, 47

Ammodramus bairdii, 12 savannarum, 12

Ammophila breviligulata, 201

Anaphalis margaritacea, 204

Andrena alleghaniensis, 300

asteroides, 300

dunningi, 300

illinoiensis, 300

milwaukeensis, 300

nasonii, 300

vicina, 300

Andromeda polifolia, 243

Andropogon hallii, 349

Anemone cylindrica, 303

Anguilla rostrata, 18

Anomodon minor, 332 rostratus, 332

Antennaria sp., 303 monocephala, 228

Anthoxanthum monticola, 241 odoratum, 204

Anthus spragueii, 12

Antrozous pallidus, 216

Aphragmus eschscholtzianus, 228

Apis mellifera, 300

Aplodontia rufa, 216

Apocynum cannabinum, 303

Apodemus semotus, 127

Aquilegia canadensis, 303

Arabis hirsuta, 303

Arctagrostis latifolia, 309

Arctic, White-veined, 357

Arctophila fulva, 234

Arctostaphylos uva-ursi, 47,299

Arenaria lateriflora, 204 serpyllifolia, 303

Aristida purpurea var. longiseta, 349

Aronia prunifolia, 204

Artemisia sp., 317 cana, 12

Ash, 237 norvegica, 228

Black, 237

Green, 317

Red, 249

Asio flammeus, 307

Asio flammeus, Ecology on Arctic Tundra, Yukon, Canada, Some Observations of Short-eared Owl, 307

Aspen, 162

Trembling, 7,22,107,249,317,328 
Aster macrophyllus, 303

Aster, 249

Arrow-leaved, 237

New England, 250

White Heath, 249

Astragalus negelecta, 303

Athalamia hyalina, 331

Augochlora aurata, 300

Augochloropis metallica, 300

Aulacomnium palustre, 332

Avens, Wood, 249

Aythya americana, 61 collaris, 61

Azalea, Alpine, 242

Badger, American, 197,216

Balsamorrhiza sp., 51

Baneberry, White, 237

Barbula convoluta, 335

Barilius nelsoni, 374

Barker, O.E., 255

Barley, Wild, 350

Bass, Largemouth, 18

Rock, 16

Smallmouth, 253

Bat, 257

Brazilian Free-tailed, 258

Pallid, 216

Spotted, 214

Bat Mortality, Consumption of Bats (Myotis spp.) by Raccoons (Procyon lotor) During an Outbreak of White-Nose Syndrome in New Brunswick, Canada: Implications for Estimates of, 257

Bats (Myotis spp.) by Raccoons (Procyon lotor) During an Outbreak of White-Nose Syndrome in New Brunswick, Canada: Implications for Estimates of Bat Mortality, Consumption of, 257

Bayberry, Northern, 201

Beachgrass, American, 201

Bear, American Black, 117,158,194

Grizzly, 216,310

Polar, 140,197,216

Bear (Ursus maritimus) Tracks by Inuit Hunters: Inter-rater Reliability and Inferences Concerning Accuracy, Interpretations of Polar, 140

Bearberry, 47

Beaver, 159

$$
\begin{aligned}
& \text { American, 194,253 } \\
& \text { Mountain, } 216
\end{aligned}
$$

Bee, 48,297

Beech, American, 58,359

Beechfern, Long, 246

Bees and Butterflies in Burned and Unburned Alvar Woodland: Evidence for the Importance of Postfire Succession to Insect Pollinator Diversity in an Imperiled Ecosystem, 297

Bellflower, Arctic, 241

Bembrops cadenati, 374 morelandi, 376 nelsoni, 374

Bendell, J., Review by, 266

Bentgrass, Carpet, 205 Northern, 241

Beslin, W., 359
Betula sp., 47

alleghaniensis, 58,237,359,363

nana-Pentaphylloides, 232

papyrifera, 237,328,359

pumila, 241

Bindweed, Field, 133

Birch, 47

Bog, 241

Paper, 237,328,359

Yellow, 58,237,359,363

Bison bison, 350

bison athabascae, 216

bison bison, 216

Bison, 350

Plains, 216

Wood, 216

Bizecki Robson, D. Rarity Status Assessments of Bugseeds (Amaranthaceae: Corispermum) in Manitoba, 338

Bladderpod, Great Plains, 349

Blue, Arctic, 357

Cranberry, 357

Northern, 357

Bluebell, 242

Blueberry, Bog, 241 Lowbush, 201

Bluebird, Eastern, 318 Mountain, 318

Bluegrass, 133 Kentucky, 203,250

Bluestem, Little, 351 Sand, 349

Boag, P.T., 140

Bobcat, 117,163,194

Bohrman, J.A., S.S. Stevens, and T.L. Serfass. Long-term Survival and Reproduction in a North American River Otter (Lontra canadensis) with an Intraperitoneal Radio-Transmitter, 252

Boloria alaskensis alaskensis, 357

bellona, 302

chariclea butleri, 357

Bombus sp., 52,301

bimaculatus, 300

impatiens, 300

melanopygus, 48

ternarius, 300

terricola, 300

Bonasa umbellus, 72

Bonasa umbellus, from Northwestern Ontario, A Brief Survey of Mycophagy in Ruffed Grouse, 72

Bos taurus, 200

Botfly Larva, Diptera: Cuterebridae, North American Deer Mouse, Peromyscus maniculatus, Consuming a Parasitizing, 65

Botrychium lunaria, 114

Bouteloua sp., 317 gracilis, 12

Boutin, S., 158

Bowles, J.M., 366

Brachyelytrum sp., 237

Brachypodium sp., 238 pinnatum, 238

sylvaticum, 235

Brachypodium sylvaticum, Poaceae), an Invasive Grass New to Ontario, Canada, Slender False Brome, 235

Brachythecium sp., 336 
acuminatum, 333

collinum, 331

laetum, 331

salebrosum, 333

Bramble, Five-leaved, 7

Branta canadensis, 62,311

Brinker, S.R., 241

British Columbia, Canada, Verification of a Forest Rating System to Predict Fisher, Martes pennanti, Winter Distribution in Sub-boreal Forests of, 7

British Columbia, Including Two Species New to the Province, Alpine Plant Range Extensions for Northern, 227

Brome, Hairy Woodland, 238

Heath False, 238

Slender False, 235

Smooth, 249

Brome (Brachypodium sylvaticum, Poaceae), an Invasive Grass New to Ontario, Canada, Slender False, 235

Bromus sp., 238

inermis, 249

pubescens, 238

tectorum, 133

Broomrape, Louisiana, 349

Brunton, D, 1

Brunton, D.F. A Tribute to Hubert ("Hue") Norman MacKenzie, 1922 2009, 76

Bryoerythrophyllum recurvirostre, 335

Bryophytes, 207

Bryum caespiticium, 333

Bryum pseudotriquetrum, 333

Bubo scandiacus, 308

Buckthorn, Common, 249 Glossy, 102

Buckwheat, Yellow, 349

Buffalo-Berry, Russett, 47

Bugseed, 338

American, 341

Hairy, 341

Hooker's, 341

Pallas', 341

Bugseeds (Amaranthaceae: Corispermum) in Manitoba, Rarity Status Assessments of, 338

Bullhead, Brown. 18

Bulrush, Alpine, 243

American, 206

Tufted, 242

Bumblebee, 48

Burke, B.L., 154

Burro, 207

Bush-honeysuckle, Northern, 237

Buteo lagopus, 308

Butterflies in Burned and Unburned Alvar Woodland: Evidence for the Importance of Postfire Succession to Insect Pollinator Diversity in an Imperiled Ecosystem, Bees and, 297

Butterflies Recorded on Flattop Mountain, Anchorage, Alaska, 357

Butterfly, 297,357

Calamagrostis rubescens, 7

Calcarius lapponicus, 308

Calidris bairdii, 308

melanotos, 308

pusilla, 308
Callaghan, C. The Importance of Natural History and The Canadian Field-Naturalist to Natural Science, 2

Callophrys henrici, 302

niphon, 302

polios, 302

Caltha leptosepala, 230

Calypso bulbosa, 52 bulbosa var. americana, 106

Calystegia sepium, 204 spithamea, 303

Campanula rotundifolia, 242,303 uniflora, 241

Campbell (1943 2011): Biologist, Conservationist, Pastor, Robert (Bob) Ronald, 370

Campylium chrysophyllum, 332 hispidulum, 332

Canada: A Preliminary Survey, Tardigrades of Fish Creek Provincial Park, Alberta, 22

Canada, A Second Amelanistic Eastern Red-backed Salamander, Plethodon cinereus, from Nova Scotia, 359

Canada Awards 2011, Alliance of Natural History Museums of, 393

Canada: Disease Management Implications, Home Range, Movements, and Habitat Utilization of Striped Skunk (Mephitis mephitis) in Scarborough, Ontario, 27

Canada, Eastern Chipmunk, Tamias striatus, Attack on a Maritime Garter Snake, Thamnophis sirtalis pallidulus, at Cooks Lake, Halifax County, Nova Scotia, 55

Canada, Evidence Confirms the Presence of Cougars (Puma concolor) in Ontario, 116

Canada, Fetid Dogweed (Dyssodia papposa; Asteraceae) and Slender Russian Thistle (Salsola collina; Amaranthaceae), New to Alberta, 366

Canada, from the Sutton Ridges, Hudson Bay Lowland Ecoregion, Additions to the Vascular Flora of Ontario, 241

Canada: Implications for Estimates of Bat Mortality, Consumption of Bats (Myotis spp.) by Raccoons (Procyon lotor) During an Outbreak of White-Nose Syndrome in New Brunswick, 257

Canada, Major Threats Facing Terrestrial Mammals in, 213

Canada, Slender False Brome (Brachypodium sylvaticum, Poaceae), an Invasive Grass New to Ontario, 235

Canada, Some Observations of Short-eared Owl, Asio flammeus, Ecology on Arctic Tundra, Yukon, 307

Canada, The Recent Spread and Potential Distribution of Phragmites australis subsp. australis in, 95

Canada, The Warty Dory, Allocyttus verrucosus New to the Fish Fauna of, 74

Canada, Two Amelanistic Eastern Red-backed Salamanders (Plethodon cinereus) from Eastern, 58

Canada, Verification of a Forest Rating System to Predict Fisher, Martes pennanti, Winter Distribution in Subboreal Forests of British Columbia, 7

Canada, Verified by Trail Camera Photographs Between 2008 and 2010, Presence of Mammals in Ontario, 193

Canadian Field-Naturalist, Advice for Contributors to The, 94

Canadian Field-Naturalist Instructions for Authors, The, 190, 294,412

Canadian Field-Naturalist Online, The, 5

Canadian Field-Naturalist, The, 1 
Canadian Field-Naturalist to Natural Science, The Importance of Natural History and The, 2

Canadian Herpetologist (TCH) 1(2), Fall 2011, The, 393

Canadian Orchids Benefit from Woodland Hiking Trails and the Implications, Some Wild, 105

Caners, R.T. Saxicolous Bryophytes of an Ordovician Dolomite Escarpment in Interlake Manitoba, with New Species Records for the Province, 327

Canis familiaris, 67

latrans, $32,117,158,194$

lupus, 67,117,158,162,194,353

lupus dingo, 132,159

lupus familiaris, 132

lupus lycaon, 216

lupus pallipes, 159

Canis lupus, Carry and Cache the Collars of Radio-collared White-tailed Deer, Odocoileus virginianus, they Killed, Wolves, 67

Canis lupus, Killing and Caching of an Adult White-tailed Deer, Odocoileus virginianus, by a Single Gray Wolf, 162

Canis lupus) Movements and Behavior Around a Kill Site and Implications for GPS Collar Studies, Gray Wolf, 353

Canis lupus, Pup Mortality: Interspecific Predation or NonParental Infanticide?, Wolf, 158

Cardamine bellidifolia, 228

Carex sp., 230,308,317

arctata, 237

bigelowii, 242

capillaris, 47

capitata, 242

deflexa, 241

gracillima, 237

gynocrates, 243

microchaeta, 227

pedunculata, 237

podocarpa, 228

pyrenaica, 229

rariflora, 244

richardsonii, 299

scirpoidea, 241

silicea, 204

Caribou, 163,197,354

Barren-ground, 216

Peary, 216

Woodland, 214

Carterocephalus palaemon skada, 357

Cassiope sp., 232

tetragona, 230

Castor canadensis, 159,194,253

Catling, P.M., 200,297

Catling, P.M. and B. Kostiuk. Some Observations on the Pollination of Round-Leaf Orchid, Galearis (Amerorchis) rotundifolia, Near Jasper, Alberta, 47

Catling, P.M. and B. Kostiuk. Some Wild Canadian Orchids Benefit from Woodland Hiking Trails and the Implications, 105

Catling, P.M. and G. Mitrow. The Recent Spread and Potential Distribution of Phragmites australis subsp. australis in Canada, 95

Catling, P.M. and M.J. Oldham. Recent Expansion of Spiranthes cernua (Orchidaceae) into Northern Ontario due to Climate Change?, 34
Catling, P.M., Reviews by, 86,277

Catostomus sp., 374 commersoni, 16

Cattail, Broadleaf, 58

Cedar, Eastern White, 107,237

Celastrina ladon ssp. lucia, 302

Celosia sp., 338

Centunculus minimus, 207

Cerastium fontanum var. vulgatum, 204

Ceratina calcarata, 300 dupla, 300

Ceratoides lanata, 12

Cercyonis pegala, 302

Cervus elaphus, 163,194

Chaetodipus hispidus, 321

Chamaesyce geyeri, 349 serpens, 133

Cheatgrass, 133

Chenopodium album, 350 quinoa, 338 subglabrum, 338

Cherry, Pin, 359

Chipmunk, Eastern, 55,154,198

Chipmunk, Tamias striatus, Attack on a Maritime Garter Snake, Thamnophis sirtalis pallidulus, at Cooks Lake, Halifax County, Nova Scotia, Canada, Eastern, 55

Choke-cherry, 249

Cinquefoil, Shrubby, 47

Circus cyaneus, 308

Cirsium discolor, 303 vulgare, 303

Cladina sp., 244 rangiferina, 204

Clark, H.O. Jr., Reviews by, 90,167

Claytonia sp., 232 sarmentosa, 228

Clethrionomys gapperi, 126

Cliffbrake, Gastony's, 327 Western Dwarf, 327

Clinopodium vulgare, 237,303

Cloudberry, 244

Clover, Hairy Prairie, 338

Clubmoss, Stiff, 244

Coad, B.W., 74

Cockscomb, 338

Coenonympha tullia inornata, 302

Colaptes auratus, 318

Colias eurytheme, 302 philodice, 302 philodice vitabunda, 357

Colletes inaequalis, 300

Coltsfoot, Palmate, 7

Conardia compacta, 332

Conium maculatum, 133

Conover, M.R., 132

Conservation and Biology of Tortoises and Freshwater Turtles Annual Symposium 2012, 282

Convolvulus arvensis, 133

Copablepharon longipenne, 338

Copper, Dorcas, 357

Corallorhiza maculata, 110 trifida, 110

Cordgrass, Marshhay, 206

Corispermum sp., 338 
americanum, 339

americanum var. americanum, 341

hookeri, 339

hookeri var. hookeri, 341

hyssopifolium, 341

nitidum, 347

pallasii, 339

villosum, 339

Corispermum) in Manitoba, Rarity Status Assessments of Bugseeds (Amaranthaceae:, 338

Cornus sericea ssp. sericea, 303 stolonifera, 249,303

Corvus brachyrhynchos, 45 corax, 45,159,308

Corydalis pauciflora, 228

Cottongrass, 308 Tussock, 243

Cottontail, Eastern, 130,194,321

Nuttall's, 216

Cottunculus nudus, 376

Cougar, 116,193 Eastern, 116

Cougars (Puma concolor) in Ontario, Canada, Evidence Confirms the Presence of, 116

Cow, 200

Coyote, $32,117,158,194$

Cranberry, 205

Crane, Sandhill, 308

Crappie, Black, 18

Creedia alleni, 376 partimsquamigera, 376

Cress, Aleutian, 228

Crow, American, 45

Crowberry, Black, 201,241

Crown-vetch, Purple, 249

Crystallodytes pauciradiatus, 376

Culaea inconstans, 374

Currant, Skunk, 246

Cuterebra americana, 66 fontinella, 66

Cycloloma atriplicifolium, 349

Cynanchum rossicum, 249

Cynomys ludovicianus, 154,216

Cyperus schweinitzii, 349 squarrosus, 349

Cypress, Summer, 350

Cypripedium acaule, 76,114 arietinum, 108,327 parviflorum var. pubescens, 108

Cystopteris fragilis, 241

Dactylorhiza sp., 51 sambucina, 53

Daisy, Oxeye, 237

Dalea villosa var. villosa, 338

Danaus plexippus, 302

Danthonia spicata, 204,237,299

Darter, Smoothbelly, 253

Dasiphora fruticosa ssp. floribunda, 47

Deer, Mule, 121 White-tailed, 67,117,162,194,239,353

Deer, Odocoileus virginianus, by a Single Gray Wolf, Canis lupus, Killing and Caching of an Adult White-tailed, 162
Deer, Odocoileus virginianus, they Killed, Wolves, Canis lupus, Carry and Cache the Collars of Radio-collared White-tailed, 67

Deermouse, 255

Delphinium sp., 232

brachycentrum, 232

chamissonis, 232

glareosum, 232

glaucum, 232

Derbowka, D., 213

Deschampsia flexuosa, 204

Descurainia sophia, 133

Dezhi, L. and Q. Aili, Reviews by, 89,270,271,274,383,384, 386,387

Diadophis punctatus, 69 punctatus edwardsii, 69

Diadophis punctatus edwardsii, on Big Tancook Island, Mahone Bay, Nova Scotia, The Brown Morph of the Northern Ringneck Snake, 69

Diapensia lapponica, 242

Diapensia, Lapland, 242

Dicranum polysetum, 299

Dicrostoynx sp., 255,311 groenlandicus, 308

Didelphis virginiana, 194

Didymodon rigidulus var. rigidulus, 331

Diervilla lonicera, 63,237,303

Dingo, 132,159

Diphascon sp., 24 granifer, 24 pingue, 24 prorsirostre, 24

Dipodomys ordii, 216

Dissostichtus eleginoides, 75

Distichium capillaceum, 333

Distichlis spicata, 133

Ditrichum flexicaule, 333

Dog, 67

African Wild, 159

Domestic, 132

Dogweed, Fetid, 366

Dogweed (Dyssodia papposa; Asteraceae) and Slender Russian Thistle (Salsola collina; Amaranthaceae), New to Alberta, Canada, Fetid, 366

Dogwood, Red-osier, 249

Dory, Warty, 74]

Dory, Allocyttus verrucosus New to the Fish Fauna of Canada, The Warty, 74

Douglas-fir, 7

Doyle, F.I, 307

Draba reptans, 349

Dreissena sp., 20

Dryas, 308

drummondii, 48

integrifolia, 232

Dryopteris expansa, 246

Duck, Ring-necked, 61

Durden, L.A. Butterflies Recorded on Flattop Mountain, Anchorage, Alaska, 357

Dyssodia papposa, 366

Dyssodia papposa; Asteraceae) and Slender Russian Thistle (Salsola collina; Amaranthaceae), New to Alberta, Canada, Fetid Dogweed, 366 
Ebinania australiae, 376 macquariensis, 376 malacocephala, 376

Echiniscus granulatus, 24

Echium vulgare, 303

Editor's Report for Volume 124 (2010), 187

Eedy 1944 2003, Dr. J (John) Wilson, 181

Eel, American, 18

Eider, Common, 41

Eider, Somateria mollissima, Collisions with Power Transmission Lines and Shortwave Communication Towers on the Tantramar Marsh in Southeastern New Brunswick, Migrant Common, 41

Elaeagnus commutata, 47

Eleocharis sp., 207

Elk, American, 163 North American, 194

Empetrum nigrum, 201,232,241

Encalypta procera, 333 rhaptocarpa, 333

Enhydra lutris, 252

Enodia anthedon, 302

Eoseristalis hirta, 48 rupium, 48

Epilobium anagallidifolium, 229 angustifolium, 232 parviflorum, 250

Epipactis helleborine, 106,237

Equus africanus asinus, 207 caballus, 200

Erethizon dorsatus, 194

Erigeron peregrinus, 232 philadelphicus, 303

Eriogonum flavum, 349

Eriophorum sp., 228 vaginatum, 243,308

Eriozona laxus, 48

Eristalis sp., 48 hirta, 48 rupium, 48

Ermine, 194,216

Erodium cicutarium, 133

Errata The Canadian Field-Naturalist 125(1): 53, 182

Erynnis icelus, 302 juvenalis, 302

lucilius, 302

persius, 48

persius $\mathrm{ssp}$. borealis, 48

Erythronium americanum, 249

Esox americanus americanus, 19 americanus vermiculatus, 19

lucius, 16,255

masquinongy, 20

niger, 16

Esox niger) in Ontario: Possible Range Expansion from New York Waters of Eastern Lake Ontario, First Occurrence of Chain Pickerel, 16

Etheostoma sp., 253

Euderma maculatum, 214

Eumetopias jubatus, 252

Eupeodes lapponicus, 48

Eurhynchium pulchellum, 333

Eurycea bislineata, 363 bislineata $\times$ cirrigera, 363
Eurycea bislineata) in Algonquin Provincial Park, Ontario, Communal Oviposition in the Northern Two-lined Salamander, 363

Euthamia graminifolia, 303

Evylaeus foxii, 304

Fagus grandifolia, 58,359

Falco peregrinus, 308

rusticolus, 311

sparverius, 321

Falcon, Peregrine, 308

Fekken, C., 140

Felis concolor, 116 concolor couguar, 116

Fenton, B., Review by, 80

Feral Horses on Vegetation of Sable Island, Nova Scotia, Effects of Feral, 200

Fern, Bracken, 237

Fragile, 241 Oak, 7

Festuca altaica, 230 rubra, 63,204

Filaree, Red-stem, 133

Fir, Balsam, 58,107,237,359

Subalpine, 7

Fisher, 7,117,194

Fisher, G., 74

Fisher, Martes pennanti, Winter Distribution in Sub-boreal Forests of British Columbia, Canada, Verification of a Forest Rating System to Predict, 7

Fissidens bryoides, 333

Fitzsimmons, J.M. The Canadian Field-Naturalist Online, 5

Flatsedge, Awned, 349 Schweinitz's, 349

Flicker, Northern, 318

Flixweed, 133

Forbes, G.J., 257

Forget-me-not, 309

Fox, Arctic, 198,308

Grey, 216

Red, 32,67,117,132,159,194,308

Swift, 216

Fragaria virginiana, 204,303 virginiana ssp. glauca, 47

Frangula alnus, 102,303

Fraxinus sp., 237

nigra, 237

pennsylvanica, 249,317

Freedman, B. 63

Freedman, B., P.M. Catling, and Z. Lucas. Effects of Feral Horses on Vegetation of Sable Island, Nova Scotia, 200

Fritillary, Mormon, 357

Mountain, 357

Purplish, 357

Frog, Boreal Chorus, 220

Western Chorus, 220

Frog (Pseudacris triseriata) Declined in Western Ottawa, Ontario?, Has the Western Chorus, 220

Frullania inflata, 332

Galearis sp., 49

rotundifolia, 47

spectabilis, 53,105 
Galearis (Amerorchis) rotundifolia, Near Jasper, Alberta, Some Observations on the Pollination of Round-Leaf Orchid, 47

Gar, Longnose, 16

Garbary, D., Review by, 174

Gavia arctica, 62 immer, 61

Gavia immer, Additional Data and Perspectives on Interspecific Aggression in the Common Loon, 61

Gaywings, 237

Gentiana platypetala, 234

Geomyces destructans, 257

Geranium bicknellii, 303

Gerard, A., Review by, 269

Geum urbanum, 249

Gibson, J.F., Review by, 168,381

Gilhen, J., 55,58,359

Gilhen, J. The Brown Morph of the Northern Ringneck Snake, Diadophis punctatus edwardsii, on Big Tancook Island, Mahone Bay, Nova Scotia, 69

Glandweed, Yellow, 63

Glandweed (Parentucellia viscosa (L.) Caruel) and Whorled Loosestrife (Lysimachia quadrifolia L.), Two Vascular Plants New to Nova Scotia: Yellow, 63

Glasswort, Virginia, 133

Glaucomys sabrinus, 55,194 volans, 55

Glaucopsyche lygdamus, 48,302

Goldenrod, Canada, 237

Rough-stemmed, 237

Seaside, 201

Tall, 249

Goodyera oblongifolia, 106 repens, 106

Goose, Canada, 62,311

Goosefoot, Smooth, 338

Gould, J., 366

Grama, Blue, 12

Granulacanthus joenelsoni, 374

Grass, False Melic, 237

Marram, 201

Poverty Oat, 237

Rabbitfoot, 133

Red Three-awned, 349

Grayling, Arctic, 255

European, 255

Grayling, Thymallus arcticus, American Pygmy Shrew, Sorex hoyi, Consumed by an Arctic, 255

Grimmia teretinervis, 331

Grothman, G.T. Tardigrades of Fish Creek Provincial Park, Alberta, Canada: A Preliminary Survey, 22

Groundsel, Yukon, 227

Grouse, Ruffed, 72

Grouse, Bonasa umbellus, from Northwestern Ontario, A Brief Survey of Mycophagy in Ruffed, 72

Grove-sandwort, Blunt-leaf, 205

Grus canadensis, 308

Gull, Glaucous, 309

Gulo gulo, 194,216,310

Gunson, K., 220

Gymnocarpium dryopteris, 7

Gymnostomum aeruginosum, 335

Gyrfalcon, 311
Halibut, Greenland, 74

Halichoerus grypus, 200

Halictus confusus, 300 ligatus, 300 rubicundus, 300

Hare, European, 194

Snowshoe, 56,194

Harrier, Northern, 308

Hawk, Rough-legged, 308

Heal-all, 237,249

Hebda, R.J., 227

Hedeoma hispida, 303

Hedge-parsley, Erect, 249

Hedwigia ciliata, 334

Hedysaum alpinum, 48

Helianthus petiolaris, 349

Helleborine, 237

Broadleaf, 108

Hemerocoetes artus, 376 morelandi, 376

Hemlock, Eastern, 359 Poison, 133

Hesperia sassacus, 302

Heydon, P.A., G.C. Miller, and M.J. Oldham. Hairy St. John'swort (Hypericum hirsutum L.) in the Toronto Area, New to North America, 248

Hieracium piloselloides, 303 scabrum 204

Hierochloe alpina, 228

Hippuris montana, 233

Honeysuckle, Bell's, 249

Hop-Hornbeam, Eastern, 237

Hoplitis pilosifrons, 300

Hordeum jubatum, 350

Hornbeam, Hop, 237

Horse, 200

Houston, C.S., Reviews by, 85,388

Houstonia sp., 51

Hoyle, J.A. and C. Lake. First Occurrence of Chain Pickerel (Esox niger) in Ontario: Possible Range Expansion from New York Waters of Eastern Lake Ontario, 16

Hudak, M., 359

Huettmann, F., Reviews by, 165,169,175,262,263,272,275

Hutchison, L.J., 72

Huynh, H.M., B.L. Burke, and D.F. McAlpine. Records of Melanistic American Red Squirrels (Tamiasciurus hudsonicus) from Nova Scotia, 154

Hylaeus illinoensis, 300

Hylocomium splendens, 299,334

Hypericum sp., 248

boreale, 207

hirsutum, 248

perforatum, 248,303

punctatum, 248

Hypericum hirsutum L.) in the Toronto Area, New to North America, Hairy St. John's-wort, 248

Hypnum vaucheri, 334

Hypsibius convergens, 24

Icegrass, 230

Ilex verticillata, 204

Imre, I., and D. Derbowka. Major Threats Facing Terrestrial Mammals in Canada, 213 
Isanthus brachiatus, 303

Isatis tinctoria, 133

Isohypsibius $\mathrm{sp} ., 24$ prosostomus, 24 lunulatus, 24 tuberculatus, 24

Isopterygiopsis pulchella, 334

Jaeger, Long-tailed, 308

Parasitic, 308

Pomarine, 307

Jaguar, 117,218

John, R., Reviews by, 82,83,84,173,261,268,279,379,380,382

Jones, S.L. Territory Size in Mixed-grass Prairie Songbirds, 12

Juncus articulatus, 207 balticus, 204

balticus var. littoralis, 203

bufonius, 207 canadensis, 207

Jung, T.S., A. Milani, O.E. Barker, and N.P. Millar. American Pygmy Shrew, Sorex hoyi, Consumed by an Arctic Grayling, Thymallus arcticus, 255

Juniper, Common, 201

Creeping, 201

Juniperus sp., 211

communis, 204,232,299

communis var. megistocarpa, 201

horizontalis, 201

Kalmia sp., 244

polifolia, 244

procumbens, 242

Kelly, P., 27

Kennedy, A.C., 41

Kenney, A.J., 307

Kestrel, American, 321

Knotweed, 133

Common, 350

Kochia scoparia ssp. scoparia, 350

Koenigia islandica, 230

Kostiuk, B., 47,105

Krebs, C.J., 307

Kronland, W.J. and M. Restani. Effects of Post-Fire Salvage Logging on Cavity-Nesting Birds and Small Mammals in Southeastern Montana, 316

Labrador Tea, Bog, 241

Marsh, 242

Lactarius sp., 72

Ladies'-tresses, Hooded, 37

Nodding, 34

Northern Slender, 108

Lady's-slipper, Greater Yellow, 108

Ram's-head, 108,327

Pink, 76

Lake, C., 16

Lamb's-quarters, 350

Lamprey, Sea, 16

Lapposyrphus lapponicus, 48

Larix laricina, 58

Larkspur, Northern, 232

Larus hyperboreus, 309

Lasioglossum divergens, 300

foxii, 300 imitatum, 300

leucozonium, 300

rufitarsis, 300

versans, 300

versatum, 300

Latham, A.D.M., and S. Boutin. Wolf, Canis lupus, Pup Mortality: Interspecific Predation or Non-Parental Infanticide?, 158

Lathyrus japonicus, 204 japonicus var. maritimus, 201

Laurel, Great, 359

Legros, D.L. Communal Oviposition in the Northern Twolined Salamander (Eurycea bislineata) in Algonquin Provincial Park, Ontario, 363

Lemming, 255

Brown, 307

Northern Collared, 308

Lemmus sp., 311 trimucronatus, 307

Leontodon autumnalis, 204

Leopard, 117

Lepisosteus osseus, 16

Lepomis gibbosus, 18 macrochirus, 20

Lepus americanus, 56,194 europaeus, 194

Leskeella nervosa, 334

Lesquerella arenosa, 349

Leucanthemum vulgare, 237

Lichen, Reindeer, 244

Ligusticum scoticum, 63

Lilium philadelphicum, 303

Limenitis archippus, 302 arthemis arthemis, 302

Limnichthys polyactis, 376

Linaria vulgaris, 204,303

Lingonberry, 241

Linnaea borealis, 204

Liparis loeselii, 38

Liverwort, 331

Loiseleuria sp., 244 procumbens, 244

Longspur, Lapland, 308

Longstaffe, F.J., 126

Lonicera tatarica, 303 bella, 249

Lontra canadensis, 198,252

Lontra canadensis) with an Intraperitoneal Radio-Transmitter, Long-term Survival and Reproduction in a North American River Otter, 252

Loon, Arctic, 62 Common, 61

Loon, Gavia immer, Additional Data and Perspectives on Interspecific Aggression in the Common, 61

Loosestrife, Bog, 206 Whorled, 63

Loosestrife (Lysimachia quadrifolia L.), Two Vascular Plants New to Nova Scotia: Yellow Glandweed (Parentucellia viscosa (L.) Caruel) and Whorled, 63

Lophocolea minor, 332

Love-lies-bleeding, 338

Lucas, Z., 200

Lupine, 309

Luzula sp., 227 confusa, 241 
multiflora, 204

spicata, 229

Lycaena dorcas arcticus, 357

Lycaon pictus, 159

Lycopodium annotinum, 244

Lycopus uniflorus, 207

Lynx canadensis, 117,163,194 rufus, $117,163,194$

Lynx, Canada, 117,163,194

Lysimachia quadrifolia, 63 terrestris, 206

Lysimachia quadrifolia L.), Two Vascular Plants New to Nova Scotia: Yellow Glandweed (Parentucellia viscosa (L.) Caruel) and Whorled Loosestrife, 63

Macdonald, M. and B. Freedman. Two Vascular Plants New to Nova Scotia: Yellow Glandweed (Parentucellia viscosa (L.) Caruel) and Whorled Loosestrife (Lysimachia quadrifolia L.), 63

MacKenzie, A. and D. Brunton. The Canadian Field-Naturalist, 1

MacKenzie, 1922 2009, A Tribute to Hubert ("Hue") Norman, 76

MacKenzie, W.H., 227

MacKinnon, C.M. and A.C. Kennedy. Migrant Common Eider, Somateria mollissima, Collisions with Power Transmission Lines and Shortwave Communication Towers on the Tantramar Marsh in Southeastern New Brunswick, 41

Macrobiotus harmsworthi, 24

Maianthemum canadense, 303 stellata, 211 stellatum, 108,204

Malloch, D., 257

Manitoba, Rarity Status Assessments of Bugseeds (Amaranthaceae: Corispermum) in, 338

Manitoba, with New Species Records for the Province, Saxicolous Bryophytes of an Ordovician Dolomite Escarpment in Interlake, 327

Mannia fragrans, 331 sibirica, 331

Maple, Red, 58,237,358 Sugar, 58,107,237,363

Marmot, Vancouver Island, 216 Yellow-Bellied, 154

Marmota flaviventris, 154 monax, 194 vancouverensis, 216

Marr, K.L., R.J. Hebda, and W.H. MacKenzie. Alpine Plant Range Extensions for Northern British Columbia, Including Two Species New to the Province, 227

Marten, American, 8,194,216

Martes americana, 8,194 americana atrata, 216 pennanti, $7,117,194$

Martes pennanti, Winter Distribution in Sub-boreal Forests of British Columbia, Canada, Verification of a Forest Rating System to Predict Fisher, 7

Mayweed, Fetid, 366

McAlpine, D.F., 154

McAlpine, D.F., K.J. Vanderwolf, G.J. Forbes, and D. Malloch. Consumption of Bats (Myotis spp.) by Raccoons (Procyon lotor) During an Outbreak of White-Nose Syndrome in New Brunswick, Canada: Implications for Estimates of Bat Mortality, 257

McLaren, I., Review by, 389
Mech, L.D., 67

Mech, L.D. Gray Wolf (Canis lupus) Movements and Behavior Around a Kill Site and Implications for GPS Collar Studies, 353

Medicago lupulina, 303

Megasyrphus laxus, 48

Megisto cymela cymela, 302

Melanerpes erythrocephalus, 318 lewis, 316

Melanoleuca sp., 72

Mephitis mephitis, 27,132,194

Mephitis mephitis) in Scarborough, Ontario, Canada: Disease Management Implications, Home Range, Movements, and Habitat Utilization of Striped Skunk, 27

Mice (Peromyscus maniculatus) in the Rocky Mountains, Alberta, Seasonal Moulting in Deer, 126

Micromys minutus, 130

Micropterus dolomieu, 253 salmoides, 18

Microtus sp., 255 californicus, 126

oеconomus, 308 pennsylvanicus, 56,321 pinetorum, 216

Milani, A., 255

Millar, J.S., 126

Millar, N.P., 255

Miller, B.M., R.J. Aitken, M.J. Oldham, and A.A. Reznicek. Slender False Brome (Brachypodium sylvaticum, Poaceae), an Invasive Grass New to Ontario, Canada, 235

Miller, G.C., 248

Milnesium sp., 25 tardigradum, 24

Minerslettuce, Bostock's, 232

Mink, American, 194

Minuartia groenlandica, 241

Minutes of the 132nd Annual Business Meeting of The Ottawa Field-Naturalists' Club January 18, 2011, 283

Mitchella repens, 204

Mitrow, G., 95

Mnium ambiguum, 334 spinulosum, 334 thomsonii, 334

Moehringia lateriflora, 205

Mole, Eastern, 216 Townsend's, 216

Montana, Effects of Post-Fire Salvage Logging on CavityNesting Birds and Small Mammals in Southeastern, 316

Montia sp., 232 bostockii, 232

Moonwort, Common, 114

Moore, J.-D. and J. Gilhen. Two Amelanistic Eastern Redbacked Salamanders (Plethodon cinereus) from Eastern Canada, 58

Moose, 194,353

Morella pensylvanica, 201

Morone americana, 18

Morris, C.J., B.W. Coad, and G. Fisher. The Warty Dory, Allocyttus verrucosus New to the Fish Fauna of Canada, 74

Mortierella sp., 258

Morton, M.L. and M.E. Pereyra. Additional Data and Perspectives on Interspecific Aggression in the Common

Moss, 331 
Spike, 12

Moth, Dusky Dune, 338

Mouse, Black-eared, 126

Brush, 130

Deer, 126,319

Eurasian Harvest, 130

Hispid Pocket, 321

House, 56

North American Deer, 65

Olive-backed Pocket, 321

Taiwan Field, 127

Western Harvest, 216,321

White-footed, 65,321

Mouse, Peromyscus maniculatus, Consuming a Parasitizing

Botfly Larva, Diptera: Cuterebridae, North American

Deer, 65

Moxostoma sp., 18

Mucor sp. 258

Murray, A.M., M.G. Sullivan, and J. Acorn. A Tribute to Joseph Schieser Nelson, 1937 2011, 373

Mus musculus, 56

Muskellunge, 20

Muskox, 353

Muskrat, 194

Mussel, Quagga, 20

Zebra, 20

Mustard, Garlic, 249

Mustela erminea, 194 erminea haidarum, 216 nivalis, 308

Myodes sp., 255 gapperi, 126

Myopsaron nelsoni, 374

Myosotis sp., 228 asiatica, 229

Myotis sp., 257 lucifugus, 257 septentrionalis, 257

Myotis spp.) by Raccoons (Procyon lotor) During an Outbreak of White-Nose Syndrome in New Brunswick, Canada: Implications for Estimates of Bat Mortality, Consumption of Bats, 257

Myrica pensylvanica, 201

Myurella julacea, 335

Naming Rights to Two Newly Discovered Lichens Auctioned Off as Fundraisers for Two B.C. Environmental Groups, 394

Neckera pennata, 335

Needle-and-thread, 12

Nelson, M.E. and L.D. Mech. Wolves, Canis lupus, Carry and Cache the Collars of Radio-collared White-tailed Deer, Odocoileus virginianus, they Killed, 67

Nelson, M.E. Killing and Caching of an Adult White-tailed Deer, Odocoileus virginianus, by a Single Gray Wolf, Canis lupus, 162

Nelson, 1937 2011, A Tribute to Joseph Schieser, 373

Neophrynichthys angustus, 376

heterospilos, 376

magnicirrus, 376

Neotoma cinerea, 321

Neovison vison, 194

New Brunswick, Canada: Implications for Estimates of Bat Mortality, Consumption of Bats (Myotis spp.) by Raccoons (Procyon lotor) During an Outbreak of WhiteNose Syndrome in, 257
New Brunswick, Migrant Common Eider, Somateria mollissima, Collisions with Power Transmission Lines and Shortwave Communication Towers on the Tantramar Marsh in Southeastern, 41

New York Waters of Eastern Lake Ontario, First Occurrence of Chain Pickerel (Esox niger) in Ontario: Possible Range Expansion from, 16

North America, Hairy St. John's-wort (Hypericum hirsutum L.) in the Toronto Area, New to, 248

North American Deer Mouse, Peromyscus maniculatus, Consuming a Parasitizing Botfly Larva, Diptera: Cuterebridae, 65

North American Ornithological Conference 2012, 181

Northeast Natural History Conference 2012, 181

Northeast Partners in Amphibian and Reptile Conservation Annual Meeting 2012, 181

Nova Scotia, Canada, A Second Amelanistic Eastern Redbacked Salamander, Plethodon cinereus, from, 359

Nova Scotia, Canada, Eastern Chipmunk, Tamias striatus, Attack on a Maritime Garter Snake, Thamnophis sirtalis pallidulus, at Cooks Lake, Halifax County, 55

Nova Scotia, Effects of Feral Horses on Vegetation of Sable Island, 200

Nova Scotia, Records of Melanistic American Red Squirrels (Tamiasciurus hudsonicus) from, 154

Nova Scotia, The Brown Morph of the Northern Ringneck Snake, Diadophis punctatus edwardsii, on Big Tancook Island, Mahone Bay, 69

Nova Scotia: Yellow Glandweed (Parentucellia viscosa (L.) Caruel) and Whorled Loosestrife (Lysimachia quadrifolia L.), Two Vascular Plants New to, 63

Nuphar lutea ssp. variegata, 206

Nuthatch, Red-breasted, 321 White-breasted, 321

Nymphalis antiopa, 302 vaualbum j-album, 302

Oak, English, 359 Red, 358

Odocoileus virginianus, 67,117,162,194,239,353

Odocoileus virginianus, by a Single Gray Wolf, Canis lupus, Killing and Caching of an Adult White-tailed Deer, 162

Odocoileus virginianus, they Killed, Wolves, Canis lupus, Carry and Cache the Collars of Radio-collared Whitetailed Deer, 67

Odontites vernus ssp. serotinus, 63

Oeneis bore mckinleyensis, 357

Oenothera parviflora, 204

Ogunbiyi, A., 359

Oldham, M.J., 34,235,248

Oldham, M.J. and S.R. Brinker. Additions to the Vascular Flora of Ontario, Canada, from the Sutton Ridges, Hudson Bay Lowland Ecoregion, 241

Oldham, M.J., J. Gould, and J.M. Bowles. Fetid Dogweed (Dyssodia papposa; Asteraceae) and Slender Russian Thistle (Salsola collina; Amaranthaceae), New to Alberta, Canada, 366

Oligoneuron album, 300

Oncorhynchus sp., 373 nerka, 373

Ondatra zibethicus, 194

Ontario, A Brief Survey of Mycophagy in Ruffed Grouse, Bonasa umbellus, from Northwestern, 72 
Ontario, Canada: Disease Management Implications, Home Range, Movements, and Habitat Utilization of Striped Skunk (Mephitis mephitis) in Scarborough, 27

Ontario, Canada, Evidence Confirms the Presence of Cougars (Puma concolor) in, 116

Ontario, Canada, from the Sutton Ridges, Hudson Bay Lowland Ecoregion, Additions to the Vascular Flora of, 241

Ontario, Canada, Slender False Brome (Brachypodium sylvaticum, Poaceae), an Invasive Grass New to, 235

Ontario, Canada, Verified by Trail Camera Photographs Between 2008 and 2010, Presence of Mammals in, 193

Ontario, Communal Oviposition in the Northern Two-lined Salamander (Eurycea bislineata) in Algonquin Provincial Park, 363

Ontario due to Climate Change?, Recent Expansion of Spiranthes cernua (Orchidaceae) into Northern, 34

Ontario, First Occurrence of Chain Pickerel (Esox niger) in Ontario: Possible Range Expansion from New York Waters of Eastern Lake, 16

Ontario?, Has the Western Chorus Frog (Pseudacris triseriata) Declined in Western Ottawa, 220

Ontario: Possible Range Expansion from New York Waters of Eastern Lake Ontario, First Occurrence of Chain Pickerel (Esox niger) in, 16

Opheodrys vernalis, 69

Opossum, Virginia, 194

Orchid, 105

Eastern Prairie Fringed, 96

Hooker's, 108

Northern Green, 108

Round-Leaf, 47

Showy, 105

Tall Northern Green, 38

Orchid, Galearis (Amerorchis) rotundifolia, Near Jasper, Alberta, Some Observations on the Pollination of Round-Leaf, 47

Orchids Benefit from Woodland Hiking Trails and the Implications, Some Wild Canadian, 105

Orchis sp., 49

mascula, 51

purpurea, 51 spitzelii, 53

Orobanche ludoviciana, 349

Orthotrichum anomalum, 335 obtusifolium, 335

Oryzopsis asperifolia, 7

Osmia atriventris, 300 proxima, 48,300

Osopsaron natalensis, 376

Ostrya virginiana, 237

Ottawa Field-Naturalists' Club Awards for 2010, Presented April 2011, The, 183

Otter, North American River, 198,252 Sea, 252

Otter (Lontra canadensis) with an Intraperitoneal RadioTransmitter, Long-term Survival and Reproduction in a North American River, 252

Ovibos moschatus, 353

Ovis aries, 200

Owl, Short-eared, 307 Snowy, 308

Owl, Asio flammeus, Ecology on Arctic Tundra, Yukon, Canada, Some Observations of Short-eared, 307
Oxyria sp., 228

digyna, 229

Packera paupercula, 300

Pagès, M., 140

Panicum dichotomiflorum, 207

Panthera onca, 117,218 pardus, 117

Papaver sp., 229 alboroseum, 228

Papilio canadensis, 302 glaucus, 48 polixenes, 302

Paramacrobiotus $c f$. richtersi, 24

Parentucellia sp., 63 viscosa, 63

Parentucellia viscosa (L.) Caruel) and Whorled Loosestrife (Lysimachia quadrifolia L.), Two Vascular Plants New to Nova Scotia: Yellow Glandweed, 63

Pascopyrum smithii, 12

Passerculus sandwichensis, 308 sandwichensis princeps, 200

Pea, Beach, 201

Pearly-everlasting, Common, 205

Pedicularis bracteosa, 232

Peeper, Spring, 222

Pellaea gastonyi, 327 glabella ssp. occidentalis, 327

Penstemon sp., 51 hirsutus, 303

Perca flavescens, 16

Perch, White, 18 Yellow, 16

Pereyra, M.E., 61

Perognathus fasciatus, 321 leucopus, 321

Peromyscus sp., 130,255 boylii, 130

leucopus, 65 maniculatus, 65,126,319 maniculatus gracilis, 65 melanotis, 126

Peromyscus maniculatus, Consuming a Parasitizing Botfly Larva, Diptera: Cuterebridae, North American Deer Mouse, 65

Peromyscus maniculatus) in the Rocky Mountains, Alberta, Seasonal Moulting in Deer Mice, 126

Petasites sp., 230 frigidus, 230

Petromyzon marinus, 16

Petsites frigidus var. palmatus, 7

Phalarope, Red-necked, 308

Phalaropus lobatus, 308

Phegopteris connectilis, 246

Phippsia sp., 231 algida, 227

Phoca sp., 354 vitulina, 200

Phragmites sp., 96 australis, 96,133 australis ssp. americanus, 95 australis ssp. australis, 95

Phragmites australis subsp. australis in Canada, The Recent Spread and Potential Distribution of, 95 
Phyciodes tharos, 302

Picea sp., 42,162

engelmanii $\times$ glauca, 7

engelmannii, 106,232

glauca, 22,47,58,106,154,158,298,328,346,359

mariana, 7,244

rubens, 58

Pickerel, Chain, 16

Grass, 19

Redfin, 19

Pickerel (Esox niger) in Ontario: Possible Range Expansion from New York Waters of Eastern Lake Ontario, First Occurrence of Chain, 16

Picoides arcticus, 316

dorsalis, 316

villosus, 318

Pieris angelika angelika, 357

oleracea, 302

rapae, 302

Pig, 200

Pigweed, Redroot, 338

Winged, 349

Pike, Northern, 16,255

Pine, Eastern White, 358

Jack, 106,162

Lodgepole, 7,106

Ponderosa, 316

Pinegrass, 7

Pinus banksiana, 106,162

contorta, 7

contorta var. latifolia, 106

ponderosa, 316

strobus, 298,359

Piperia unalascensis, 110

Pipit, Sprague's, 12

Plagiochila porreloides, 332

Plagiomnium cuspidatum, 334 medium, 334

Plantago lanceolata, 204 major, 207 maritima, 63

Plantain, Menzies' Rattlesnake, 107

Platanthera sp., 53

aquilonis, 38,108

hookeri, 108

hyperborea, 38

leucophaea, 96

Platydictya jungermannioides, 334

Plebejus glandon bryanti, 357

idas, 357

idas alaskensis, 357

optilete, 357

optilete yukona, 357

Plestiodon septentrionalis, 338

Plethodon cinereus, 58,359

Plethodon cinereus) from Eastern Canada, Two Amelanistic Eastern Red-backed Salamanders, 58

Plethodon cinereus, from Nova Scotia, Canada, A Second Amelanistic Eastern Red-backed Salamander, 359

Plover, American Golden, 308

Pluvialis dominica, 308

Poa sp., 133,230

alpina, 228

аппиа, 204

paucispicula, 230 pratensis, 203,250

Poanes hobomok, 302

Pohlia cruda, 333

Polargrass, 309

Polemonium sp., 228

Polites mystic, 302

themistocles, 302

Polygala paucifolia, 237

senega, 303

Polygonatum pubescens, 237

Polygonia comma, 302

faunus, 302

interrogationis, 302

progne, 302

Polygonum sp., 133

aviculare ssp. depressum, 350

hydropiper, 207

hydropiperoides, 206

viviparum, 228

monspeliensis, 133

Pomoxis nigromaculatus, 18

Pond-lily, Variegated Yellow, 206

Pondweed, Broadleaf, 206

Claspingleaf, 206

Small, 206

Poplar, Balsam, 22

Poppy, Pale, 228

Populus balsamifera, 22 tremuloides, 7,22,107,162,249,298,317,328

Porcupine, North American, 194

Potamogeton pectinata, 206 perfoliatus, 206 pusillus ssp. tenuissimus, 206

Potentilla sp., 232

biflora, 232

diversifolia, 228

elegans, 233

norvegica, 303

Power, M., 27

Prairie Dog, Black-Tailed, 154,216

Procyon lotor, 32,117,132,194,257

Procyon lotor, During an Outbreak of White-Nose Syndrome in New Brunswick, Canada: Implications for Estimates of Bat Mortality, Consumption of Bats (Myotis spp.) by Raccoons, 257

Proulx, G. Verification of a Forest Rating System to Predict Fisher, Martes pennanti, Winter Distribution in Subboreal Forests of British Columbia, Canada, 7

Prunella vulgaris, 237,249,303

Prunus pensylvanica, 359 virginiana, 249,299

Pseudacris crucifer, 222

maculata, 220

triseriata, 220

Pseudacris triseriata) Declined in Western Ottawa, Ontario?, Has the Western Chorus Frog, 220

Pseudoleskeella tectorum, 334

Pseudoroegneria sp, 317

Pseudotsuga menziesii, 7

Psychrolutes microporos, 376 sio, 376

Pteridium aquilinum, 237

Pteropsaron heemstrai, 376

Puma concolor, 116,193 concolor couguar, 116 
Puma concolor) in Ontario, Canada, Evidence Confirms the Presence of Cougars, 116

Pungitius pungitius, 374

Pylaisiella polyantha, 334

Quercus robur, 359 rubra, 359

Quinoa, 338

Raccoon, 32,132,194,257

Common, 117

Raccoons (Procyon lotor) During an Outbreak of White-Nose Syndrome in New Brunswick, Canada: Implications for Estimates of Bat Mortality, Consumption of Bats (Myotis spp.) by, 257

Radula complanata, 332

Ramazzottius oberhaeuseri, 24

Rangifer tarandus, 163,198,354

tarandus caribou, 214

tarandus groenlandicus, 216

tarandus pearyi, 216

Ranunculus sp., 230

eschscholtzii, 229

flammula, 207

pygmaeus, 230

repens, 207

Rat, Ord's Kangaroo, 216

Rattlesnake-plantain, Dwarf, 106

Raven, Common, 45,159,308

Redhead, 61

Redhorse, 18

Reed, Common, 95,133

Reid, D.G., F.I. Doyle, A.J. Kenney, and C.J. Krebs. Some Observations of Short-eared Owl, Asio flammeus, Ecology on Arctic Tundra, Yukon, Canada, 307

Reinhardtius hippoglossoides, 74

Reithrodontomys megalotis, 321 megalotis dychei, 216 megalotis megalotis, 216

Renaud, C.B. Robert (Bob) Ronald Campbell (1943 2011): Biologist, Conservationist, Pastor, 370

Restani, M., 316

Reznicek, A.A., 235

Rhamnus cathartica, 249,303 frangula, 102

Rhinanthus sp., 63 crista-galli, 204

Rhodobryum ontariense, 333

Rhododendron groenlandicum, 241 lapponicum, 241

maximum, 359

tomentosum, 242

Rhus aromatica, 303 rydbergii, 303 typhina, 303

Ribes glandulosum, 246

Ricegrass, Indian, 349 Rough-leaved, 7

Rosa sp., 300 acicularis, 303

blanda, 303

virginiana, 204

virginiana var. virginiana, 201

Rosatte, R. Evidence Confirms the Presence of Cougars (Puma concolor) in Ontario, Canada, 116
Rosatte, R., P. Kelly and M. Power. Home Range, Movements, and Habitat Utilization of Striped Skunk (Mephitis mephitis) in Scarborough, Ontario, Canada: Disease Management Implications, 27

Rosatte, R. Presence of Mammals in Ontario, Canada, Verified by Trail Camera Photographs Between 2008 and 2010, 193

Rose, Virginia, 201

Rosebay, Lapland, 241

Rosemary, Bog, 243

Rubus sp., 51,304

arcuans, 204

chamaemorus, 244

idaeus ssp. strigosus, 303

odoratus, 303

pedatus, 7

Rumex acetosella, 204

Rush, Baltic, 205

Russell, R.W., W. Beslin, M. Hudak, A. Ogunbiyi, A. Withrow, and J. Gilhen. A Second Amelanistic Eastern Redbacked Salamander, Plethodon cinereus, from Nova Scotia, Canada, 359

Russula sp., 72

Ruzicka, R.E., and M.R. Conover. Influence of Wind and Humidity on Foraging Behavior of Olfactory Mesopredators, 132

Sagina procumbens, 207

Salamander, Eastern Red-backed, 58,358

Northern Two-lined, 363

Salamander (Eurycea bislineata) in Algonquin Provincial Park, Ontario, Communal Oviposition in the Northern Two-lined, 363

Salamander, Plethodon cinereus, from Nova Scotia, Canada, A Second Amelanistic Eastern Red-backed, 359

Salamanders (Plethodon cinereus) from Eastern Canada, Two Amelanistic Eastern Red-backed, 58

Salicornia virginica, 133

Salix sp., 22

arctica, 232

glauca, 312

polaris, 227

pulchra, 312

reticulata, 228

richardsonii, 308

stolonifera, 228

vestita, 241

Salmo sp., 373

trutta, 255

Salmon, Kokanee, 373

Salsola sp., 366

collina, 366

kali ssp. kali, 366

tragus, 338,366

Salsola collina; Amaranthaceae), New to Alberta, Canada, Fetid Dogweed (Dyssodia papposa; Asteraceae) and Slender Russian Thistle, 366

Saltgrass, Inland, 133

Saltwort, Common, 366

Sander vitreus, 16

Sander-Regier, R., Reviews by, 81,90,265

Sandmat, Matted, 133

Sandpiper, Baird's, 308

Pectoral, 308

Semipalmated, 308 
Sanguisorba canadensis, 232

Sarcobatus vermiculatus, 12

Saxifraga bronchialis, 232

$$
\text { ferruginea, } 230
$$

lyallii, 230

nelsoniana, 228

rivularis, 244

rivularis ssp. arctolitoralis, 246

rivularis ssp. rivularis, 246

Saxifrage, Alpine Brook, 244

Scalopus aquaticus, 216

Scapanus townsendii, 216

Schistidium frigidum, 331

Schizachne purpurascens, 237

Schizachyrium scoparium, 351

Schoenoplectus americanus, 206

Scirpus americanus, 206

Sciurus carolinensis, 55,154,194 niger, 154

Scutellaria parvula, 303

Seal, 354

Gray, 200

Harbor, 200

Sea Lion, California, 252

Steller, 252

Seburn, D., Reviews by, 172,382

Seburn, D.C. and K. Gunson. Has the Western Chorus Frog (Pseudacris triseriata) Declined in Western Ottawa, Ontario?, 220

Securigera varia, 249

Sedge, 308

Bigelow's, 242

Capitate, 242

Drooping Woodland, 237

Graceful, 237

Hair-Like, 47

Long-stalked, 237

Loose-flowered Alpine, 244

Northern, 241

Northern Bog, 243

Northern Single-spike, 241

Selaginella densa, 12,327

Seligeria donniana, 331

Senecio triangularis, 232 yukonensis, 228

Serfass, T.L., 252

Sheep, 200

Shepherdia canadensis, 7,47,303

Shinnersoseris rostrata, 349

Shorthusk, 237

Shrew, 255

American Pygmy, 255

Common, 255

Dusky, 255

Masked, 255

Pacific Water, 216

Shrew, Sorex hoyi, Consumed by an Arctic Grayling, Thymallus arcticus, American Pygmy, 255

Sialia currucoides, 318 sialis, 318

Silene acaulis, 229 antirrhina, 303

Silver-Berry, American, 47

Sisyrinchium montanum, 303

Sitta canadensis, 321 carolinensis, 321

Skeletonweed, Annual, 349

Skink, Prairie, 338

Skipperling, Arctic, 357

Skunk, Striped, 27,132,194

Skunk (Mephitis mephitis) in Scarborough, Ontario, Canada: Disease Management Implications, Home Range, Movements, and Habitat Utilization of Striped, 27

Slipper, Fairy, 106

Smartweed, Swamp, 206

Smith, H., 140

Snake, Eastern Smooth Green, 69

Garter, 56

Maritime Garter, 55,69

Northern Redbelly, 56,69

Ringneck, 69

Snake, Diadophis punctatus edwardsii, on Big Tancook Island, Mahone Bay, Nova Scotia, The Brown Morph of the Northern Ringneck, 69

Snake, Thamnophis sirtalis pallidulus, at Cooks Lake, Halifax County, Nova Scotia, Canada, Eastern Chipmunk, Tamias striatus, Attack on a Maritime Garter, 55

Solidago sp., 300

altissima, 249

canadensis, 237,250,303

juncea, 303

nemoralis, 300

rugosa, 204,237

sempervirens, 201

Solomon's Seal, False, 108

Hairy, 237

Somateria mollissima, 41 mollissima dresseri, 41

Somateria mollissima, Collisions with Power Transmission Lines and Shortwave Communication Towers on the Tantramar Marsh in Southeastern New Brunswick, Migrant Common Eider, 41

Soopalie, 7

Sorex sp., 255

araneus, 255

bendirii, 216

cinereus, 255

hoyi, 255

monticolus, 255

Sorex hoyi, Consumed by an Arctic Grayling, Thymallus arcticus, American Pygmy Shrew, 255

Sorrel, Common Sheep, 205

Sparrow, Baird's, 12

Grasshopper, 12

Ipswich, 200

Savannah, 200,308

Spartina patens, 206 pectinata, 204

Spermophilus parryii, 154 tridecemlineatus, 154,321

Speyeria aphrodite, 302 cybele, 302 mormonia bischoffii, 357

Sphagnum sp., 58,207,232

Sphagnum, 58

Spikemoss, Prairie, 327

Spinach, 338

Spinacia oleracea, 338

Spiranthes cernua, 34 lacera, 108 
romanzoffiana, 37

Spiranthes cernua (Orchidaceae) into Northern Ontario due to Climate Change?, Recent Expansion of, 34

Spruce, 42,162

Black, 7,244

Engelmann's, 106

Red, 58

White, 7,22,47,58,106,154,158,328,346,359

Spurge, Geyer's, 349

Squirrel, American Red, 154

Arctic Ground, 154

Eastern Fox, 154

Eastern Gray, 55,154,194

Gray, 154

Northern Flying, 55,194

Red, 55,154,194

Southern Flying, 55

Thirteen-lined Ground, 154,321

Squirrels (Tamiasciurus hudsonicus) from Nova Scotia, Records of Melanistic American Red, 154

St. John's-wort, 248

Common, 248

Hairy, 248

Spotted, 248

St. John's-wort (Hypericum hirsutum L.) in the Toronto Area, New to North America, Hairy, 248

Starling, European, 318

Stellaria graminea, 204

Stercorarius longicaudus, 308

parasiticus, 308

pomarinus, 307

Stevens, S.S., 252

Stewart, F.E.C. North American Deer Mouse, Peromyscus maniculatus, Consuming a Parasitizing Botfly Larva, Diptera: Cuterebridae, 65

Stewart, 1936-2011, Dr. Kenneth William, 282

Stickleback, Brook, 374

Ninespine, 374

Stipa comata, 12

Stitchwort, Greenland, 241

Storeria occipitomaculata occipitomaculata, 56

Strawberry, 47 Virginia, 205

Streptopus amplexifolius, 7

Stuckenia pectinata, 206

Sturnus vulgaris, 318

Sucker, White, 16

Sullivan, M.G., 373

Sulphur, Clouded, 357

Sunfish, Bluegill, 20 Pumpkinseed, 18

Sunflower, Prairie, 349

Sus scrofa, 200

Swallow-wort, European, 249

Sweetgrass, Alpine, 241

Sylvilagus floridanus, 130,194,321 nuttallii nuttallii, 216

Symphyotrichum sp., 249 albus, 299

ciliolatum, 303

ericoides, 249

lanceolatum, 250

novae-angliae, 250

urophyllum, 237

novi-belgii, 204

Syntrichia norvegica, 331 ruralis, 331

Tabacaru, C.A., J.S. Millar, and F.J. Longstaffe. Seasonal Moulting in Deer Mice (Peromyscus maniculatus) in the Rocky Mountains, Alberta, 126

Tadarida brasiliensis, 258

Tamarack, 58

Tamarisk, 133

Tamarix sp., 133

Tamias striatus, 55,154,198

Tamias striatus, Attack on a Maritime Garter Snake, Thamnophis sirtalis pallidulus, at Cooks Lake, Halifax County, Nova Scotia, Canada, Eastern Chipmunk, 55

Tamiasciurus hudsonicus, 55,154,194

Tamiasciurus hudsonicus) from Nova Scotia, Records of Melanistic American Red Squirrels, 154

Tanney, J.B. and L.J. Hutchison. A Brief Survey of Mycophagy in Ruffed Grouse, Bonasa umbellus, from Northwestern Ontario, 72

Taraxacum officinale, 207,303

Taxidea taxus, 197 taxus jacksoni, 216 taxus jeffersonii, 216

Taxus canadensis, 359

Taylor, A.N. and P.M. Catling. Bees and Butterflies in Burned and Unburned Alvar Woodland: Evidence for the Importance of Postfire Succession to Insect Pollinator Diversity in an Imperiled Ecosystem, 297

Tephroseris sp., 228

frigida, 228

kjellmanii, 228

lindstroemii, 228

tundricola, 228

yukonensis, 227

Tetropium fuscum, 359

Thalictrum pubescens, 204

Thamnophis sirtalis, 56 sirtalis pallidulus, 55,69

Thamnophis sirtalis pallidulus, at Cooks Lake, Halifax County, Nova Scotia, Canada, Eastern Chipmunk, Tamias striatus, Attack on a Maritime Garter Snake, 55

Thistle, Prickly Russian, 366

Russian, 338,350

Slender Russian, 366

Thistle (Salsola collina; Amaranthaceae), New to Alberta, Canada, Fetid Dogweed (Dyssodia papposa; Asteraceae) and Slender Russian, 366

Thorybes pylades, 302

Thuidium recognitum, 335

Thuja occidentalis, 107,237,298

Thymallus arcticus, 255 thymallus, 255

Thymallus arcticus, American Pygmy Shrew, Sorex hoyi, Consumed by an Arctic Grayling, 255

Thymelicus lineola, 302

Toothfish, Patagonian, 75

Torilis japonica, 249

Tortella fragilis, 332 tortuosa, 335

Tortula mucronifolia, 335

Toxicodendron rydbergii, 303

Tragopogon dubius, 303

Trichophorum alpinum, 243 cespitosum, 242

Trientalis borealis, 204

Trifolium sp., 51 
aureum, 63

hybridum, 303

repens, 204

Trisetum sp., 228 spicatum, 232

Troglodytes aedon, 320

Trout, Brown, 255

Trout-lily, Yellow, 249

Tsuga canadensis, 359

Tundrius sp., 312

Turbot, 74

Tussock, 309

Twayblade, Loesel's, 38

Twistedstalk, Clasping-leaved, 7

Typha sp., 96 latifolia, 58,100

Urocyon cinereoargenteus, 216

Ursus americanus, 117,158,194 arctos, 216,310 maritimus, 140,197,216

Ursus maritimus) Tracks by Inuit Hunters: Inter-rater Reliability and Inferences Concerning Accuracy, Interpretations of Polar Bear, 140

Vaccinium angustifolium, 201 macrocarpon, 204

uliginosum, 241 vitis-idaea, 241

Van Coeverden de Groot, P., 140

Vanderwolf, K.J., 257

Vanessa cardui, 302

Verbascum thapsus, 303

Verbena bracteata, 349

Vervain, Large-bracted, 349

Vetch, 308 Tufted, 250

Viburnum nudum, 204

Vicia cracca, 250

Vine, Dog-strangling, 249

Viola adunca, 303 labradorica, 237

lanceolata, 207

Violet, 237 nephrophylla, 303

Vole, California, 126 Meadow, 56,321

Southern Red-backed, 126

Tundra, 308

Woodland, 216

Vulpes lagopus, 198,308

velox, 216

vulpes, 32,67,117,132,159,194,308

Waldsteinia fragarioides, 303

Wallengrenia egeremet, 302
Walleye, 16

Weasel, Least, 308

Wheatgrass, Crested, 133,350

Western, 12

White, Arctic, 357

Whitlow-grass, Carolina, 349

Wild-basil, 237

Willow, 22,312

Arctic, 308

Hairy, 241

Richardson's, 308

Willowherb, Small-flowered, 250

Withrow, A., 359

Woad, Dyer's, 133

Wolf, 67,117,158

Eastern, 216

Gray, 158,162,194,353

Indian, 159

Wolf, Canis lupus, Killing and Caching of an Adult Whitetailed Deer, Odocoileus virginianus, by a Single Gray, 162

Wolf (Canis lupus) Movements and Behavior Around a Kill Site and Implications for GPS Collar Studies, Gray, 353

Wolf, Canis lupus, Pup Mortality: Interspecific Predation or Non-Parental Infanticide?, 158

Wolverine, 194,216,310

Wolves, Canis lupus, Carry and Cache the Collars of Radiocollared White-tailed Deer, Odocoileus virginianus, they Killed, 67

Wong, P.B.Y., P. Van Coeverden de Groot, C. Fekken, H. Smith, M. Pagès, and P.T. Boag. Interpretations of Polar Bear (Ursus maritimus) Tracks by Inuit Hunters: Inter-rater Reliability and Inferences Concerning Accuracy, 140

Woodchuck, 194

Woodfern, Spreading, 246

Woodpecker, American Three-toed, 316

Black-backed, 316

Hairy, 318

Lewis's, 316

Red-headed, 318

Woodrat, Bushy-tailed, 321

Woodrush, Northern, 241

Woodsia ilvensis, 241

Woodsia, Rusty, 241

World Congress of Herpetology 7, 181

Wren, House, 320

Yarrow, Common, 201

Yew, Canada, 359

Youngman: 1927 2011, Phillip Merrill, 394

Yukon, Canada, Some Observations of Short-eared Owl, Asio flammeus, Ecology on Arctic Tundra, 307

Zalophus californianus, 252 


\section{Index to Book Reviews}

\section{Botany}

Daoming, G. Coastal Wetland Ecosystem Spartina Salt Marsh and Its Management in China, 89

Dong, P. and L. Xinzheng. Walnut Germplasm Resources in China, 386

Guohang, T. and Z. Tianbang. Cactus Plant Resources and Utilization, 385

Hudson, P. A Field Guide to Alpine Flowers of the Pacific Northwest, 173

Hudson, R. A Field Guide to Gemstones of the Pacific Northwest, 173

Lindeberg, M.R. and S.C. Lindstrom. Field Guide to the Seaweeds of Alaska, 174

Liuyan, Y.Y. and X. Lin. Outbreak, Harm and Control of Algal Blooms in Lakes of China, 270

Riley, J. Wetlands of the Hudson Bay Lowlands: An Ontario Review, 173

Sharma, J. North American Native Orchid Conservation Preservation, Propagation, and Restoration, 86

\section{Environment}

Benson, E. Wired Wilderness: Technologies of Tracking and the Making of Modern Wildlife, 90

Morse, S. Sustainability: A Biological Perspective, 90

\section{Other}

Dickinson, E.C., L.K. Overstreet, R.J. Dowsett and M.D. Bruce. !Priority! The Dating of Scientific Names in Ornithology: A Directory to the Literature and its Reviewers, 390

Liao B., M. Li, Y. Chen, W. Guan. Techniques for Restoration and Reconstruction of Mangrove Forests in China, 389

Pauly, D. 5 Easy Pieces - The Impact of Fisheries on Marine Ecosystems, 275

Rice, S.A. Life of Earth - Portrait of A Beautiful, MiddleAged, Stressed-Out World, 274

Riley, J.L. Wetlands of the Ontario Hudson Bay Lowland: A Regional Overview, 277

Riley, J. Wetlands of the Ontario Hudson Bay Lowlands: An Ontario Review, 279

Rustamov, E. and A. Rustamov. Biodiversity Conservation in Central Asia: On the Example of Turkmenistan, 175

Shucheng, X., Y. Hongfu, S. Xiaoying. Interactions and Coevolution of Life and Earth Environment, 388

UNESCO Publishing. Climate Change and Arctic Sustainable Development: Scientific, Social, Cultural and Educational Challenges, 272

Watson, A. A Zoologist on Baffin Island - 1953, 391

Zhiyun, O. and Z. Chunquan. Atlas of Biodiversity and Conservation in the Yangtze River Basin, 271

\section{Zoology}

Alexander, B. Natural History. The Ultimate Visual Guide to Everything on Earth, 84

Arlott, N. Birds of North America and Greenland, 261

Brown, B.V., A. Borkent, J.M. Cumming, D.M. Wood, N.E. Woodley, and M.A. Zumbado. Manual of Central American Diptera, Volumes 1 and 2, 168

Bavington, D. Managed Annihilation: An Unnatural History of the Newfoundland Cod Collapse, 262

Belgrano, A. and C.W. Fowler. Ecosystem Based Management for Marine Fisheries: An Evolving Perspective, 263

Coonan, T.J., C.A. Schwemm, and D.K. Garcelon, with L. Munson and C. Asa. Decline and Recovery of the Island Fox: A Case Study for Population Recovery, 265

Crossley, R. The Crossley ID Guide: Eastern Birds, 82

del Hoyo, J., A. Elliott, and D.A. Christie. Handbook of the Birds of the World. Volume 15. Weavers to New World Warblers, 83

del Hoyo, J., A. Elliott and D. Christie. Handbook of Birds of the World. Volume 16, 381

Dunn, J.L. and J. Alderfer. National Geographic Field Guide to the Birds of North America Sixth Edition, 382

Ernst, C.H. and E.M. Ernst. Venomous Reptiles of the United States, Canada, and Northern Mexico. Volume 2. Crotalus, 384

Gibson, D. and G.V. Byrd. Birds of the Aleutian Islands, 165

Hill, G.E. Bird Color Mysteries Explained, 82

Howell, S.N.G. Petrels, Albatrosses and Storm Petrels of North America, 384

Hunter, L. Carnivores of the World, 382

Jackson, D.C. Life in a Shell: A Physiologist's View of a Turtle, 172

Kenefick, M., R. Restall, and F. Hayes. Field Guide to the Birds of Trinidad and Tobago, 261

Knick, S.T. and J.W. Connelly. Greater Sage-Grouse, Ecology and Conservation of a Landscape Species and Its Habitats, 266

McDaniel, N. A Field Guide to Sea Stars of the Pacific Northwest, 269

Packer, L. Keeping the Bees: Why All Bees Are at Risk and What We Can Do to Save Them, 81

Price, P.W., R.F. Denno, M.D. Eubanks, D.L. Finke, and I. Kaplan. Insect Ecology: Behavior, Populations and Communities, 383

Richardson, P. Bats, 80

Scott, G. Essential Ornithology, 85

Stokes, D.W. and L.Q. Stokes. The Stokes Field Guide to the Birds of North America, 167

Wilson, D.E. and R.A. Mittermeier. Handbook of the Mammals of the World. Volume 2. Hoofed Mammals, 268

Woodley, K. Godwits: Long-haul Champions, New Zealand to the World One Bird's Voyage, 169 
